\section{Territórios do Sistema Único de Saúde - mapeamento das redes de atenção hospitalar}

\author{
The territorial basis of the Brazilian National \\ Health System: mapping hospital networks
}

Evangelina X. G. de Oliveira 1

Marilia Sá Carvalho 2

Cláudia Travassos 3

\section{Introdução}

1 Coordenação de Geografia, Fundação Instituto Brasileiro de Geografia e Estatística, Rio de Janeiro, Brasil.

2 Departamento de Epidemiologia e Métodos Quantitativos em Saúde, Escola Nacional de Saúde Pública, Fundação Oswaldo Cruz, Rio de Janeiro, Brasil.

3 Departamento de Informações em Saúde, Centro de Informação Científica e Tecnológica Fundação Oswaldo Cruz, Rio de Janeiro, Brasil.

Correspondência Evangelina X. G. de Oliveira Coordenação de Geografia. Fundação Instituto Brasileiro de Geografia e Estatística. Av. República do Chile 500, Rio de Janeiro, $R J$ 20031-070, Brasil. evan@iis.com.br

\section{Abstract}

This article presents two types of networks organized according to patient caseload in health services, concerning both primary hospital care (most frequent hospital procedures) and tertiary care (high-cost procedures). Data on inpatient care in Brazil in 2000 obtained from the Hospital Information System of the Unified National Health System were aggregated by place of residence and hospital location at the municipal level. Both the network structure and the node (municipality) hierarchy were established using the dominant flow approach. In addition, a typology of flows was applied to indicate the degree of connection across the networks. Primary hospital care networks reach most of the country, and few municipalities are not connected to the network. Relatively few cities provide higher-level services, and almost half of the municipalities are unconnected. The Ministry of Health aims to provide access to frequently used health services near the users' place of residence, and this goal appears to be feasible in the short run. On the other hand, much remains to be done to ensure widespread access to tertiary care.

Health Services Accessibility; Attention; Health Services
O mapeamento das redes estabelecidas pelo deslocamento das pessoas que buscam atendimento pelo Sistema Único de Saúde (SUS), em todo o Brasil, permite identificar lacunas na distribuição dos serviços. Um quadro de referência nacional, que permita situar os estudos mais detalhados, é útil para investigar em que medida o SUS avançou na promoção da universalização do acesso. De fato, para que os serviços possam ser acessíveis a todos, o planejamento do setor saúde precisa conhecer a sua distribuição e a de seus usuários nas várias escalas, da escala local à nacional.

A regionalização e a hierarquização da rede de serviços de saúde são diretrizes do SUS. Até recentemente, a estratégia de descentralização, caracterizada pela municipalização, recebeu maior destaque do que a hierarquização dos serviços. Com a Norma Operacional de Atenção à Saúde (NOAS-SUS), publicada em janeiro de 2001, foi retomada a proposta original de hierarquização através da organização de redes articuladas e efetivas de serviços que integrem as capacidades de diversos municípios, de modo a alcançar economias de escala, e evitar ineficiências no sistema 1 .

Na geografia, redes são estruturas de interconexão, constituídas por dois tipos de elementos: pontos (ou nós) e as ligações entre eles. A configuração das ligações revela a estrutura da rede 2 . 
Nas redes territoriais, os lugares correspondem aos pontos, e as ligações podem ser materiais, como as estradas, ou imateriais, como os sinais eletromagnéticos 3 . O estudo das redes perpassa vários campos do conhecimento, e adquiriu particular relevância nos últimos anos.

As redes configuram-se em árvore, ou em malha 3 . Redes em árvore, também conhecidas como redes hierárquicas (ou piramidais), caracterizam-se pela limitação das conexões entre seus diversos ramos. Predominam os fluxos hierárquicos, de um centro menor para o seu superior e, não havendo caminhos alternativos, podem ocorrer pontos de estrangulamento, impedindo o acesso da população aos níveis superiores da hierarquia. Já as redes organizadas em malha - em que cada nó se liga a vários outros - permitem percorrer caminhos variados entre os pontos, de modo que os diversos ramos da rede estão interconectados.

Na Saúde Pública, o conceito de acesso é complexo, e expressa o grau de ajuste entre as necessidades dos usuários e a oferta de serviços de saúde, segundo a idéia inicialmente proposta por Donabedian ${ }^{4}$. Neste trabalho, acesso assume uma definição mais restrita, a de acessibilidade geográfica, já referida por Donabedian. Segundo essa conceituação inicial, acessibilidade é um dos componentes do acesso, aquele que relaciona a localização da oferta e a localização dos usuários, considerados os meios de transporte destes e o tempo, a distância e os custos envolvidos no deslocamento 5,6. O nível de renda das pessoas condiciona a intensidade com que o uso de um serviço de saúde é afetado pela distância a que está localizado o serviço 7, e isso ocorre mesmo nos casos em que o serviço é gratuito, na medida em que o sistema de saúde afirma a universalidade, mas não garante transporte.

As barreiras representadas pela distância têm impacto diferenciado de acordo com o nível de complexidade dos serviços demandados; assim, os deslocamentos em busca de serviços especializados serão, normalmente, mais longos do que aqueles que demandam níveis de atenção mais simples 8,9. A investigação das desigualdades no acesso aos serviços de saúde deve, portanto, levar em conta o padrão de localização destes serviços, e a variação nas distâncias que os indivíduos com problemas de saúde semelhantes devem percorrer para obter atendimento.

O presente trabalho investiga as redes estabelecidas, no Brasil, para diferentes tipos de de- manda de serviços de saúde - mais simples e mais complexos. Dois tipos de rede foram investigados: a configurada pela atenção hospitalar básica, definida pelas condições de maior freqüência de internação e as redes estabelecidas por tratamentos de alta complexidade, para examinar variações de escala e direcionamento dos fluxos que demandam serviços mais especializados.

\section{Material e métodos}

Este estudo examinou as internações pagas pelo SUS - no ano de 2000 em todo o Brasil - agregadas segundo o município de internação e o de residência dos pacientes. Os dados foram obtidos no Sistema de Informações Hospitalares do SUS (SIH-SUS), considerando-se apenas as Autorizações de Internação Hospitalar (AIH) de tipo 1 (registros que, em geral, correspondem às internações). O total de AIH do tipo 1 foi de 11.937.323; e destas, 2.953 .531 (24,7\%) foram realizadas em município diferente do município de residência dos pacientes.

Para investigar a organização da rede de atenção hospitalar básica, o total das internações no Brasil foi desagregado, por idade, em dois grupos: o das pessoas com cinco anos e mais de idade (10.234.858 AIH) e o das crianças com idade menor do que cinco anos (1.702.465 AIH). Com esta divisão, buscou-se avaliar as mudanças na extensão e na configuração das redes provocadas pela menor capacidade de deslocamento das crianças. Selecionou-se as internações de maior freqüência, tomando como referência a variável "procedimento realizado" (Tabela de Procedimentos) 10, que é a unidade de pagamento do SUS. Para o primeiro grupo incluiu-se apenas os quarenta procedimentos mais freqüentes, responsáveis, no mínimo, por $0,5 \%$ das internações no país, relacionados na Tabela 1, e correspondendo a 6.417.217 internações. Uma vez identificado o subconjunto de procedimentos, selecionou-se as internações em que o município de residência do paciente era diferente do município de sua internação, encontrando-se 1.377.224 nesta situação. Aplicou-se a mesma seqüência e critérios de seleção para gerar o grupo composto pelas internações das crianças, um total de 1.442 .583 internações, das quais 291.962 ocorreram fora de seu município de residência. Para essa faixa etária, são trinta os procedimentos mais freqüentes, responsáveis, no mínimo, por $0,5 \%$ das inter- 
Identificação dos procedimentos mais freqüentes - população com cinco e mais anos de idade. Brasil, 2000.

\begin{tabular}{|c|c|c|c|}
\hline Código & Descrição & $\%$ no Brasil & $\begin{array}{l}\% \text { fora do } \\
\text { domicílio }\end{array}$ \\
\hline 35001011 & Parto normal & 11,7 & 7,6 \\
\hline 35021012 & Parto normal com atendimento do recém-nascido na sala de parto & 4,3 & 2,5 \\
\hline 77500113 & Insuficiência cardíaca & 3,8 & 2,9 \\
\hline 63001403 & Tratamento em psiquiatria em hospital psiquiátrico - b & 3,3 & 5,7 \\
\hline 35009012 & Cesariana & 3,2 & 2,4 \\
\hline 35014016 & Curetagem pós-aborto & 2,3 & 1,5 \\
\hline 35025018 & Parto normal - exclusivamente para hospitais amigos da criança & 2,2 & 1,7 \\
\hline 76500225 & Doença pulmonar obstrutiva crônica & 2,2 & 1,3 \\
\hline 74500252 & Entero infecções (clínica médica) & 2,1 & 1,0 \\
\hline 81500106 & AVC agudo & 1,8 & 1,4 \\
\hline 76500128 & Crise asmática & 1,8 & 0,8 \\
\hline 76500071 & Broncopneumonia & 1,6 & 0,9 \\
\hline 35022019 & Cesariana com atendimento do recém-nascido na sala de parto & 1,6 & 1,1 \\
\hline 76500063 & Pneumonia não especificada & 1,6 & 0,9 \\
\hline 77500121 & Crise hipertensiva & 1,4 & 0,8 \\
\hline 80500072 & Pielonefrite & 1,2 & 0,7 \\
\hline 72500000 & Diagnóstico e/ou primeiro atendimento em clínica médica & 1,2 & 0,9 \\
\hline 33011117 & Herniorrafia inguinal (unilateral) & 1,2 & 1,1 \\
\hline 82500053 & Diabetes sacarino & 1,1 & 0,7 \\
\hline 34008020 & Colpoperineoplastia anterior e posterior & 1,0 & 1,0 \\
\hline 33004080 & Colecistectomia & 0,8 & 0,9 \\
\hline 75500124 & Hemorragias digestivas & 0,8 & 0,7 \\
\hline 75500035 & Gastrite e duodenite & 0,8 & 0,4 \\
\hline 35026014 & Cesariana - exclusivamente para hospitais amigos da criança & 0,8 & 0,7 \\
\hline 77500032 & Insuficiência coronariana aguda & 0,7 & 0,7 \\
\hline 80500218 & Outras afecções do aparelho gênito urinário & 0,7 & 0,5 \\
\hline 76500233 & Insuficiência respiratória aguda & 0,7 & 0,5 \\
\hline 33005060 & Apendicectomia & 0,7 & 0,6 \\
\hline 74300270 & Entero infecções (pediatria) & 0,6 & 0,3 \\
\hline 76300102 & Crise asmática & 0,6 & 0,3 \\
\hline 76500047 & Pneumonia bacteriana & 0,6 & 0,4 \\
\hline 85500879 & Intercorrências clínicas de paciente oncológico & 0,6 & 0,9 \\
\hline 76300080 & Broncopneumonia & 0,5 & 0,3 \\
\hline 33016119 & Laparotomia exploradora & 0,5 & 0,6 \\
\hline 73500011 & Desnutrição (clínica médica) & 0,5 & 0,3 \\
\hline 34010033 & Histerectomia total & 0,5 & 0,5 \\
\hline 75500272 & Colecistite aguda & 0,5 & 0,3 \\
\hline 74500201 & Estreptococcias (clínica médica) & 0,5 & 0,3 \\
\hline 80500110 & Cólica nefrética & 0,5 & 0,3 \\
\hline \multirow[t]{2}{*}{76300072} & Outras pneumonias & 0,5 & 0,3 \\
\hline & Total & 62,7 & 46,6 \\
\hline
\end{tabular}


nações (Tabela 2). Note-se que apenas quatro procedimentos integram as duas relações.

No caso das redes de alta complexidade, selecionaram-se as internações relativas aos procedimentos hospitalares de alta complexidade, definidos na Portaria SAS n. 96, de 27 de março de 2000, sendo essas internações agregadas segundo especialidades, com base na Tabela de Compatibilidade entre o Procedimento Realizado e o Diagnóstico Principal Informado da Portaria SAS n. 579, de 28 de dezembro de 2001. Dentre as 56 especialidades constantes desta tabela foram analisadas as duas responsáveis pelo maior número de internações, a saber, Cirurgia Cardíaca, com 51.695 internações, das quais 29.590 ocorreram fora do município de residência, e Neurocirurgia - Alta Complexida- de, com 94.039 internações, 41. 638 das quais em outro município.

Não foram considerados os fluxos intermunicipais representados por uma única internação, visando reduzir problemas de flutuação aleatória, o que, no final, resultou em trabalhar-se com 1.358.635 internações de pessoas com cinco anos e mais de idade e 284.655 de crianças, para a atenção hospitalar básica, e com 26.725 internações para Cirurgia Cardíaca e 38.991 para Neurocirurgia.

A unidade geográfica de análise foi o município. Em 3.640 municípios, dos 5.507 existentes no Brasil em 2000, ocorreram internações pagas pelo SUS. Na entrada dos dados dos formulários da AIH não é incomum, nos casos de ocorrência de municípios homônimos, o erro

Tabela 2

Identificação dos procedimentos mais freqüentes - crianças com menos de cinco anos de idade. Brasil, 2000.

\begin{tabular}{|c|c|c|c|}
\hline Código & Descrição & $\%$ no Brasil & $\begin{array}{l}\% \text { fora do } \\
\text { domicílio }\end{array}$ \\
\hline 76400271 & Entero infecções em lactente & 10,5 & 8,9 \\
\hline 76400085 & Broncopneumonia em lactente & 10,2 & 8,2 \\
\hline 76300102 & Crise asmática & 9,3 & 6,9 \\
\hline 74300270 & Entero infecções (pediatria) & 7,5 & 5,0 \\
\hline 76400077 & Pneumonia do lactente & 7,2 & 6,8 \\
\hline 76300080 & Broncopneumonia & 7,1 & 4,7 \\
\hline 76300072 & Outras pneumonias & 5,0 & 3,9 \\
\hline 71300015 & Prematuridade & 2,9 & 4,0 \\
\hline 76300188 & Insuficiência respiratória aguda & 2,5 & 2,5 \\
\hline 71300007 & Diagnóstico e/ou primeiro atendimento em clínica pediátrica & 2,3 & 2,2 \\
\hline 76300021 & Laringotraqueobronquite & 2,0 & 1,3 \\
\hline 72300019 & Desidratação aguda (pediatria) & 1,8 & 1,5 \\
\hline 74300261 & Septicemia (pediatria) & 1,7 & 3,0 \\
\hline 71300082 & Icterícias neonatais & 1,5 & 1,4 \\
\hline 71300112 & Outras afecções do recém-nascido & 1,5 & 1,5 \\
\hline 76300056 & Bronquiolite aguda & 1,4 & 1,2 \\
\hline 33011117 & Herniorrafia inguinal (unilateral) & 1,3 & 1,8 \\
\hline 74300237 & Estafilococcias (pediatria) & 1,1 & 1,2 \\
\hline 74300229 & Estreptococcias (pediatria) & 1,0 & 0,9 \\
\hline 71300066 & Pneumopatias agudas & 1,0 & 1,0 \\
\hline 76300064 & Pneumonia estafilocóccica & 0,8 & 0,9 \\
\hline 81300042 & Epilepsias & 0,8 & 0,9 \\
\hline 31004105 & Postectomia (circuncisão) & 0,7 & 0,8 \\
\hline 71300090 & Infecções perinatais & 0,7 & 0,7 \\
\hline 80300073 & Pielonefrite & 0,6 & 0,6 \\
\hline 33015112 & Herniorrafia umbilical & 0,5 & 0,6 \\
\hline 71300031 & Anoxia perinatal grave & 0,5 & 0,6 \\
\hline 31000002 & Cirurgia múltipla & 0,5 & 0,6 \\
\hline 71300058 & Síndrome da A.P.I. do recém-nascido (membrana hialina) & 0,5 & 0,7 \\
\hline \multirow[t]{2}{*}{73300012} & Desnutrição protéico calórica (1ㅇ e 2o graus) pediatria & 0,5 & 0,5 \\
\hline & Total & 84,9 & 74,8 \\
\hline
\end{tabular}


de identificação da Unidade da Federação do município de residência do paciente internado. Encontrou-se, por exemplo, pacientes internados em São Paulo, e residentes em Santo André, na Paraíba, bem como internações, em Cuiabá, de residentes em Várzea Grande, no Piauí, ou ainda, residentes em Caxias, no Maranhão, internados em Nova Iguaçu, no Rio de Janeiro. Tais erros prejudicam a análise dos fluxos. Para corrigi-los, selecionou-se para revisão - e quando necessário, realocou-se - os registros referentes a municípios de residência com problemas de homônimos, num total de 263 casos, representando no mínimo duas e no máximo 197 AIH. No caso dos procedimentos de alta complexidade, entretanto, não foram realizadas correções, pois, além de registrar-se apenas nove casos de dúvidas provocadas por homônimos, variando de 2 a $67 \mathrm{AIH}$, é perfeitamente plausível que pacientes residentes em São Bernardo, Maranhão, por exemplo, tenham sofrido cirurgia cardíaca em São Paulo.

A estrutura espacial pode ser investigada empregando conceitos da teoria dos grafos, ramo da topologia desenvolvido a partir do estudo de Euler, de 1736, sobre as pontes de Königsberg. Nos grafos lineares os objetos (lugares) são representados por vértices, e as ligações por arestas 13. Para identificar redes baseadas no consumo de serviços de saúde empregou-se, neste trabalho, o método do fluxo dominante, proposto por Nystuen \& Dacey 14 , que estabelece a hierarquia dos nós da rede com base em três propriedades dos fluxos: “(1) uma cidade é independente se o seu maior fluxo se dirige para uma cidade menor do que ela, $e$ subordinada se o fluxo vai para uma cidade maior; (2) transitividade: se A é subordinada a $B$ e B é subordinada a $C$, então A é subordinada a C; e (3) uma cidade não pode ser subordinada a qualquer de suas subordinadas" 15 (p. 149).

Para cada uma das redes investigadas, a matriz de fluxos entre os municípios é composta pelas células que representam o município de residência e o de internação, e pelo número de internações. Para aplicar a primeira propriedade dos fluxos, empregou-se como indicador de tamanho do município o Valor Total (em Reais) das AIH (de todos os tipos) pagas pelo SUS no ano de 2000, em cada município.

O método do fluxo dominante define, simultaneamente, o arcabouço da rede e os níveis hierárquicos dos municípios que constituem os nós. Os municípios independentes constituem o primeiro nível; os que lhes são diretamente subordinados são de nível 2; aqueles que se ligam a um município de nível 2 são de nível 3, e assim sucessivamente. Os municí- pios subordinados, diretamente ou por transitividade, constituem a rede do município de nível superior, podendo-se examinar redes ligadas a centros (municípios) de qualquer nível. Note-se que lugares de um mesmo nível hierárquico não precisam apresentar características semelhantes. Considere-se, por exemplo, uma rede formada por alguns poucos municípios, em área de povoamento rarefeito, eoutra composta por um grande centro que polariza um elevado número de municípios: o centro de mais alto nível em cada uma das redes ocupa idêntica posição (o nível 1) em ambas as redes, mantendo-se essa correspondência entre os demais níveis, apesar das diferenças de tamanho, equipamento ou funções desempenhadas pelos centros em cada uma das áreas.

$\mathrm{O}$ arcabouço traçado pelas principais ligações entre os nós (o fluxo dominante) evidencia a estrutura hierárquica da rede, mas vários outros fluxos, além do principal, estruturam os relacionamentos internos e externos de cada rede e limitar o exame apenas aos fluxos dominantes representa perda de informação. Uma forma de avaliar a diversidade de fluxos é classificá-los segundo tipologia que os distingue da seguinte forma: os hierárquicos, que acompanham a principal ligação, e os transversais, que ocorrem entre diferentes ramos, ou sub-redes (Figura 1). Além disso, é possível verificar se tais fluxos obedecem à hierarquia da rede (ascendentes) ou se são "contra-hierárquicos" (descendentes). Note-se que os fluxos hierárquicos e contra-hierárquicos podem dirigir-se para seu superior ou inferior imediato, ou podem ser capturados por um centro de outro nível, sendo então chamados de fluxos em "curto circuito" 16 . Ao considerar também as ligações existentes entre os diversos ramos da rede, além das relações de subordinação hierárquica, esta classificação permite avaliar o grau de "fechamento" ou, ao contrário, de interconexão das várias redes.

Como primeira aproximação ao exame das barreiras representadas pela distância, calculou-se a proporção de internações segundo faixas de distância. Para a identificação da rede e classificação dos fluxos, utilizou-se um programa desenvolvido por Mabini \& Rabino 17. A distância em linha reta, da sede de cada município para a de seu superior, foi calculada pelo programa ArcView, o mesmo usado para mapear as redes.

\section{Resultados}

No Brasil, em 2000, os municípios com hospital conveniado ao SUS concentravam $91,0 \%$ da 


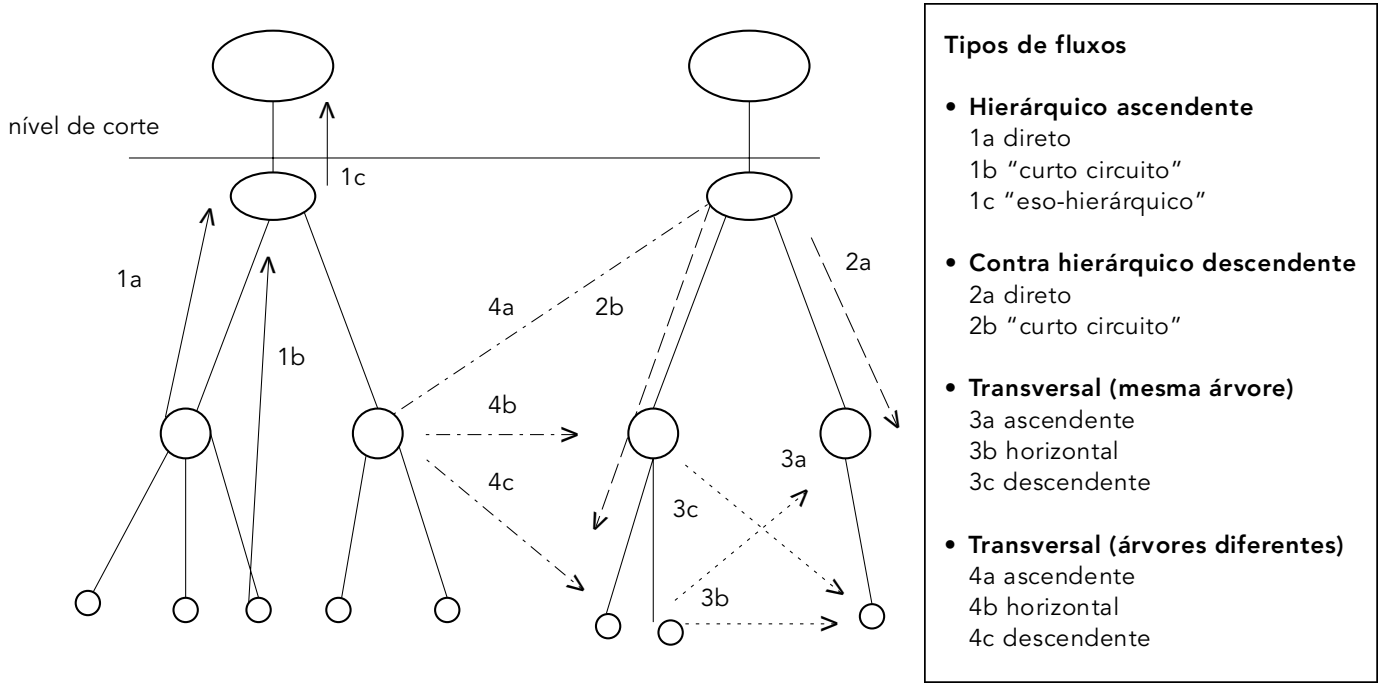

população total do país e cerca de $75,0 \%$ das internações ocorreram no município de residência do paciente. Na investigação da rede de atenção hospitalar básica, para o grupo da população de cinco anos e mais de idade verificou-se que os quarenta procedimentos mais freqüentes (Tabela 1) foram responsáveis por $62,7 \%$ das internações custeadas pelo SUS no ano 2000, em todo o país. Considerando apenas as internações realizadas em hospitais fora do município de residência do paciente, esta proporção cai para 46,6\%. No total, $2.808 \mathrm{mu}$ nicípios internaram pessoas residentes fora de seus limites. Considerando a distribuição de pacientes em todos os pares município de residência-município de internação, 35,0\% dos fluxos correspondem a menos de cinco pessoas, e $26,0 \%$ a trinta ou mais pessoas; em relação ao total acumulado de pacientes que saem de cada município, esses valores são de $0,5 \%$ e $94,0 \%$, respectivamente.

As internações de pessoas de cinco anos e mais de idade conformaram 12 redes, cinco das quais compostas por um município de nível 1 e pelo menos um subordinado. Sete municípios não enviaram pacientes para internação fora de seu território e são, por definição, centros independentes. Destes sete, três localizam-se no Amazonas, e os outros quatro, um no Maranhão, um no Piauí, um no Rio Grande do Sul e um no Mato Grosso. Note-se que, em quatro destes municípios, não há hospitais conveniados ao SUS.

As cinco redes com subordinados têm dimensões bem diversas. As duas menores são a de Rio Crespo (Rondônia) comandando apenas um município, e a de Ponte Branca (Mato Grosso) com dois municípios. Recife e Porto Alegre comandam redes com 486 e 466 municípios, respectivamente, e a maior de todas as redes é a de São Paulo, com 4.543 municípios. O mapa da Figura 2 apresenta os três primeiros níveis das maiores redes, desconsiderando os centros que não têm municípios subordinados As áreas de atuação dessas redes são marcadas por grandes diferenças de tamanho, seja em termos de área, seja em número de municípios, seja em volume de população (Tabela 3 ).

A rede comandada por Recife está organizada em sete níveis, e é composta por municípios de Pernambuco, Paraíba e Alagoas. No segundo nível contam-se 42 centros, 18 dos quais têm municípios subordinados. Entre as redes comandadas pelos centros de nível 2 destacam-se as de João Pessoa e Maceió. A classificação dos fluxos originados nos municípios da rede de Recife indica uma predominância dos fluxos transversais, que alcançam $65,2 \%$ do total, sendo 52,5\% ascendentes. Quanto aos fluxos hierárquico-ascendentes, 16,5\% são diretos, e 15,1\% em "curto circuito", saltando estágios, e indo diretamente para um nível superior. 
Figura 2

Redes de atenção hospitalar básica. População de 5 anos e mais de idade.

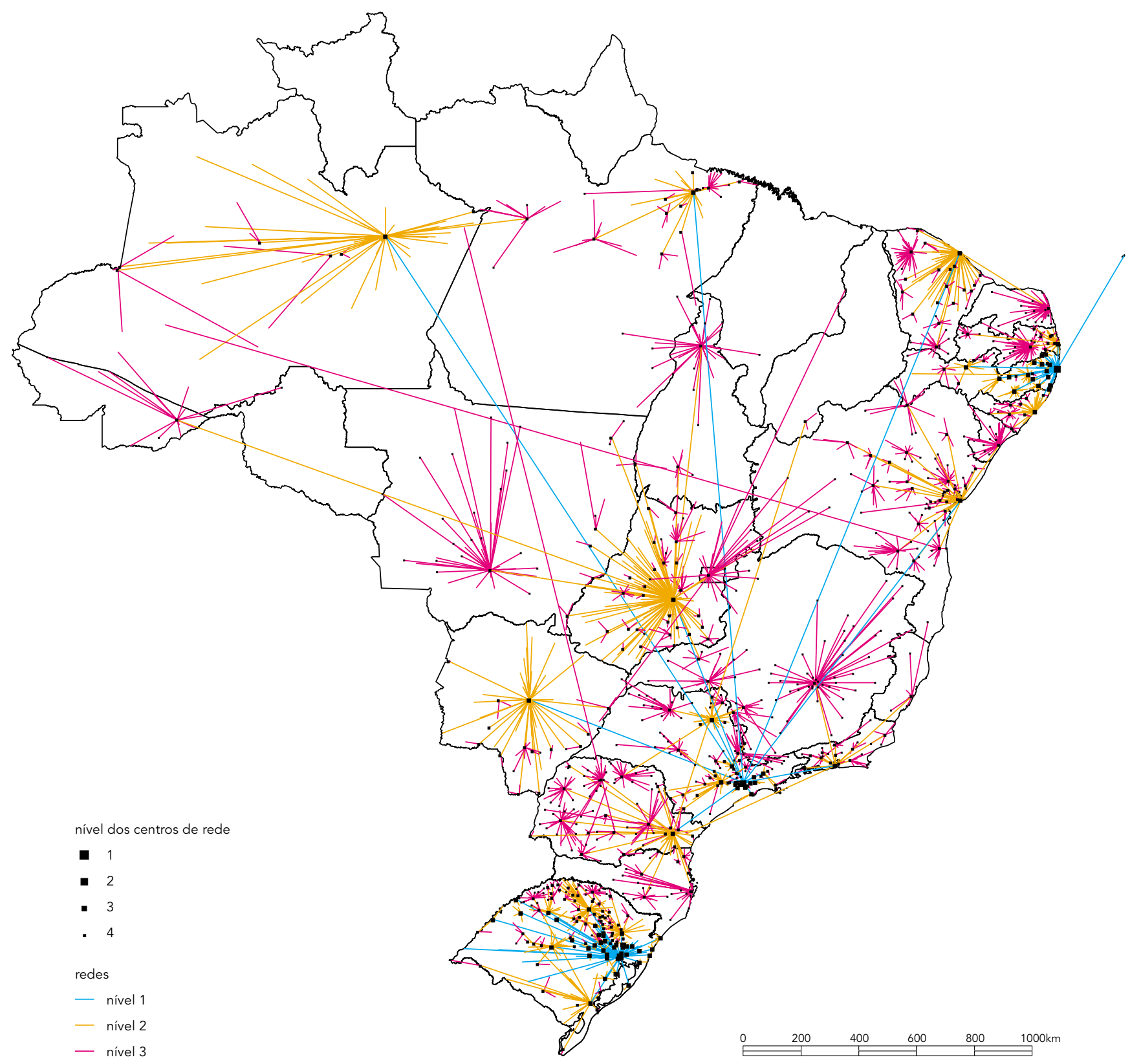


Atenção hospitalar básica (população de cinco anos e mais de idade) - principais redes de nível 1

e respectivas sub-redes de nível 2.

\begin{tabular}{|c|c|c|c|c|}
\hline Redes & $\begin{array}{l}\text { Número de } \\
\text { municípios }\end{array}$ & Área $\left(\mathbf{k m}^{2}\right)$ & $\begin{array}{c}\text { População } 2000 \\
\text { (número de habitantes) }\end{array}$ & $\begin{array}{l}\text { Número } \\
\text { de níveis }\end{array}$ \\
\hline São Paulo & 4.543 & 7.979 .070 & 145.885 .897 & 9 \\
\hline Goiânia & 1136 & 3.488 .970 & 24.734 .289 & 9 \\
\hline Rio de Janeiro & 861 & 533.282 & 32.246 .552 & 8 \\
\hline Curitiba & 616 & 283.487 & 13.306 .448 & 8 \\
\hline Salvador & 447 & 458.190 & 14.015 .884 & 7 \\
\hline Ribeirão Preto & 406 & 242.248 & 7.771 .167 & 8 \\
\hline Fortaleza & 359 & 208.038 & 10.448 .109 & 8 \\
\hline Sorocaba & 255 & 117.483 & 6.081 .255 & 8 \\
\hline Campinas & 187 & 60.436 & 7.735 .253 & 8 \\
\hline Belém & 87 & 538.714 & 4.304 .791 & 6 \\
\hline Campo Grande & 68 & 322.001 & 1.922 .952 & 5 \\
\hline Manaus & 67 & 1.711 .032 & 3.373 .388 & 6 \\
\hline São José dos Campos & 12 & 5.767 & 1.075 .890 & 4 \\
\hline Santos & 9 & 2.402 & 1.474 .665 & 4 \\
\hline Recife & 486 & 156.900 & 13.398 .680 & 7 \\
\hline João Pessoa & 218 & 53.857 & 3.442 .105 & 7 \\
\hline Maceió & 90 & 23.569 & 2.624 .537 & 5 \\
\hline Serra Talhada & 39 & 36.572 & 759.684 & 5 \\
\hline Caruaru & 22 & 9.577 & 820.772 & 4 \\
\hline Garanhuns & 22 & 6.626 & 490.929 & 3 \\
\hline Palmares & 14 & 3.600 & 393.859 & 3 \\
\hline Arcoverde & 10 & 10.647 & 269.108 & 3 \\
\hline Porto Alegre & 466 & 267.669 & 10.178 .418 & 5 \\
\hline Caxias do Sul & 116 & 36.195 & 1.526 .746 & 5 \\
\hline Passo Fundo & 87 & 26.373 & 839.074 & 5 \\
\hline Pelotas & 57 & 74.637 & 1.461 .306 & 5 \\
\hline Santa Maria & 34 & 28.298 & 564.647 & 4 \\
\hline ljuí & 30 & 9.776 & 294.340 & 5 \\
\hline Torres & 8 & 1.251 & 62.528 & 3 \\
\hline
\end{tabular}

A rede de Porto Alegre é basicamente restrita à área do Rio Grande do Sul, e seus municípios distribuem-se por cinco níveis. Apenas um município gaúcho, na fronteira com Santa Catarina, está fora dela, e um município catarinense integra a rede. No nível 2 estão 73 municípios, 41 dos quais têm subordinados. Destacam-se, no segundo nível, as redes comandadas por Caxias do Sul, Passo Fundo e Pelotas. A classificação dos fluxos originados nos municípios da rede de Porto Alegre indica uma predominância dos fluxos transversais, que alcançam 58,0\% do total, sendo $49,8 \%$ ascendentes. Quanto aos fluxos hierárquico-ascendentes, $22,7 \%$ são diretos, e 17,1\% em "curto circuito".
Estruturada em nove níveis, a rede de São Paulo é composta pelas redes dos centros de nível 2: capitais estaduais, centros regionais paulistas, e municípios da região metropolitana de São Paulo. Das redes de nível 2, a mais extensa é a de Goiânia, com 123 centros de nível 3, dos quais $85(69,1 \%)$ não apresentam ramificação própria. A dimensão da área é relativizada pela rarefação das ligações mais extensas. Note-se que, no quarto nível desta rede, subordinada a Brasília, está a sub-rede de Teresina, que engloba a de São Luís, no quinto nível. A segunda maior rede em número de municípios é aquela ligada ao Rio de Janeiro, com 18 centros de nível 3, dos quais apenas cinco $(27,8 \%)$ não têm subordinados. Destacam-se, 
entre as sub-redes do Rio de Janeiro, aquelas comandadas por Belo Horizonte (Minas Gerais) e Vitória (Espírito Santo). As redes de Fortaleza (Ceará), Campo Grande (Mato Grosso do Sul), Ribeirão Preto (São Paulo), Manaus (Amazonas) e Santos (São Paulo) têm, como Goiânia (Goiás), a maior parte dos centros de nível 3 sem ramificação, configurando hierarquização truncada, enquanto Salvador (Bahia) tem padrão mais semelhante ao do Rio de Janeiro, com melhor distribuição dos municípios pelos vários níveis. As regiões dos demais centros estão a meio caminho entre os dois tipos.

Como mencionado inicialmente, as redes podem superpor-se num mesmo território. Neste contexto observe-se, na Figura 2, o entrecruzamento das redes paulistas, e conseqüente padrão de descontinuidade territorial. Vale notar que a área de influência de Ribeirão Preto apresenta-se descontínua, capturando a rede de Londrina, no Paraná.

A classificação dos fluxos originados nos municípios da rede de São Paulo indica uma predominância dos fluxos transversais, que alcançam $60,5 \%$ do total, sendo $51,1 \%$ ascendentes. Quanto aos fluxos hierárquico ascendentes, 17,9\% são diretos, e 17,0\% em "curto circuito".

Nos 5.495 municípios abaixo do primeiro nível, 58\% das pessoas internadas residem a menos de $30 \mathrm{~km}$ do município do hospital, e $22,4 \%$ num raio de 30 a $60 \mathrm{~km}$.

A segunda rede investigada foi a de atenção hospitalar básica estabelecida a partir do subconjunto de internações limitado às crianças. Os trinta procedimentos mais freqüentes ( Tabela 2) foram responsáveis por $84,9 \%$ das internações, e por $74,8 \%$ das internações realizadas em hospital localizado fora do município de residência dos pacientes. No total, 2.140 municípios internaram pessoas residentes fora de seus limites. Considerando a distribuição de pacientes em todos os pares município de residência-município de internação, 40,0\% dos fluxos correspondem a menos de cinco pessoas, e $15,0 \%$ a trinta ou mais pessoas; em relação ao total acumulado de pacientes que saem de cada município, esses valores são de 7,0\% e 41,0\%, respectivamente.

Das 216 redes de nível 1 encontradas, 131 são compostas de um único município. Entre os municípios de nível 1, 177 não enviaram pacientes para internação fora de seu território e destes, 38 municípios não têm hospital conveniado ao SUS. A distribuição destes 38 segundo as regiões mostra que 12 estão na região Norte, 13 no Nordeste, sete no Sudeste, dois no Sul e quatro no Centro Oeste. A Figura 3 apresenta as redes dos níveis 1,2 e 3 .
Apesar do grande número de pequenas redes, verifica-se que as vinte maiores redes (Tabela 4) comandam quase $85,0 \%$ do total de municípios brasileiros, correspondendo a cerca de 90,0\% da população do país.

A maior das redes é a de Goiânia, em cuja área de influência encontram-se municípios dos Estados de Goiás, Mato Grosso, Rondônia, Pará, Tocantins, Maranhão, Bahia, e Minas Gerais, e que está estruturada em sete níveis. No segundo nível contam-se 106 centros, dos quais $68(64,2 \%)$ não apresentam ramificação própria. Entre as redes comandadas pelos centros de nível 2 destacam-se as de Brasília (Distrito Federal), Araguaína e Palmas no Tocantins.

A segunda maior rede é aquela ligada a Belo Horizonte, com municípios de Minas Gerais, Bahia e Espírito Santo (além de um município amazonense). Conta com 58 centros de nível 2, dos quais apenas 19 (32,8\%) não têm subordinados. Destacam-se, entre as sub-redes de Belo Horizonte, aquelas comandadas por Montes Claros, Teófilo Otoni e Governador Valadares.

A rede de Recife (Pernambuco) comanda municípios de Pernambuco, da Paraíba e de Alagoas, alcançando ainda alguns municípios do Rio Grande do Norte e da Bahia. Nesta rede existem 54 centros de nível 2, 35 (64,8\%) dos quais não apresentam ramificação própria. As maiores redes de nível 2 são as de João Pessoa (Paraíba) e Maceió (Alagoas).

A rede de Porto Alegre está basicamente limitada ao Rio Grande do Sul, alcançando apenas quatro municípios catarinenses, e metade dos 74 centros de nível 2 tem subordinados.

As redes de Fortaleza, Ribeirão Preto, Natal (Rio Grande do Norte), Aracaju (Sergipe), Londrina (Paraná), Bauru (São Paulo), São José do Rio Preto (São Paulo), Vitória, Curitiba (Paraná) e Campinas (São Paulo) têm, como Goiânia (Goiás), a maior parte dos centros de nível 2 sem ramificação, configurando hierarquização truncada, enquanto Rio de Janeiro, Salvador, Teresina (Piauí), Florianópolis (Santa Catarina), São Paulo e Belém (Pará) têm padrão mais semelhante ao de Belo Horizonte, com melhor distribuição dos municípios pelos vários níveis.

Na classificação dos fluxos que ocorrem nas redes de nível 1, predominam as relações hierárquicas, sendo $41,5 \%$ do tipo hierárquico ascendente direto e $14,9 \%$ em "curto circuito" Os fluxos transversais, entre ramificações da rede, somam 40,6\%; sendo $27,4 \%$ deles ascendentes.

Nos 5.290 municípios abaixo do primeiro nível, $61,1 \%$ das pessoas internadas residem a menos de $30 \mathrm{~km}$ do município do hospital, e $22,4 \%$ num raio de 30 a $60 \mathrm{~km}$. 
Redes de atenção hospitalar básica. Crianças de até 5 anos de idade.

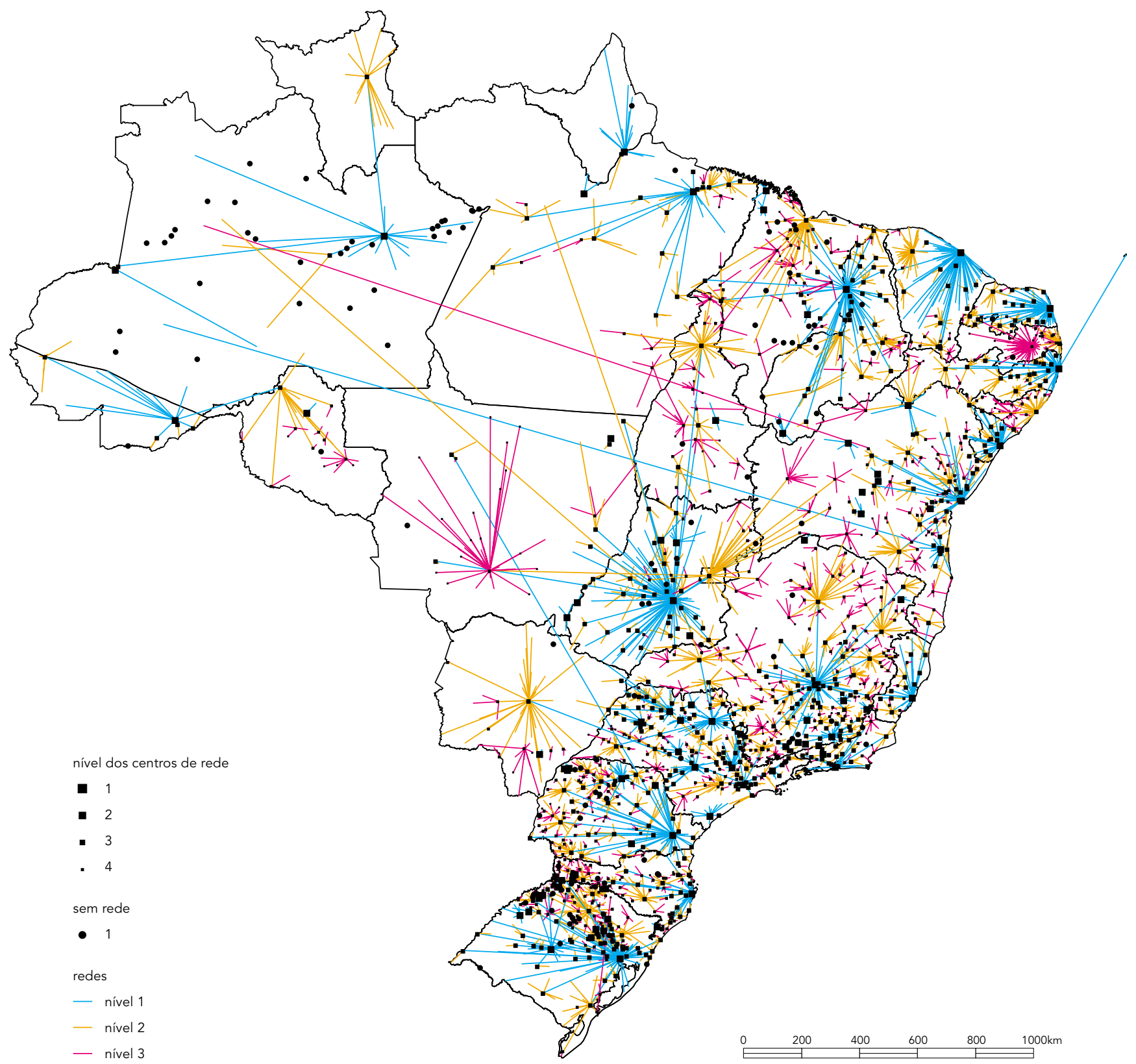


Atenção hospitalar básica (crianças) - maiores redes de nível 1.

\begin{tabular}{|c|c|c|c|c|}
\hline Rede & $\begin{array}{l}\text { Número de } \\
\text { municípios }\end{array}$ & Área $\left(\mathrm{km}^{2}\right)$ & $\begin{array}{c}\text { População } 2000 \\
\text { (número de habitantes) }\end{array}$ & Níveis \\
\hline Goiânia & 546 & 1.887 .615 & 12.026 .160 & 7 \\
\hline Belo Horizonte & 529 & 386.644 & 12.245 .397 & 6 \\
\hline Recife & 486 & 158.210 & 13.363 .282 & 7 \\
\hline Porto Alegre & 373 & 214.011 & 9.164 .190 & 6 \\
\hline Teresina & 364 & 440.683 & 7.537 .766 & 5 \\
\hline Curitiba & 305 & 205.180 & 8.595 .328 & 6 \\
\hline Salvador & 240 & 268.625 & 8.649 .580 & 5 \\
\hline Florianópolis & 227 & 72.411 & 4.050 .290 & 5 \\
\hline São Paulo & 221 & 343.523 & 24.965 .352 & 6 \\
\hline Ribeirão Preto & 206 & 203.250 & 5.430 .578 & 6 \\
\hline Fortaleza & 184 & 147.872 & 7.457 .412 & 6 \\
\hline Natal & 164 & 52.046 & 2.786 .694 & 4 \\
\hline Campinas & 115 & 35.683 & 5.586 .974 & 6 \\
\hline Belém & 110 & 866.974 & 5.458 .582 & 5 \\
\hline Aracaju & 109 & 49.794 & 2.505 .439 & 5 \\
\hline Londrina & 105 & 38.399 & 1.807 .322 & 5 \\
\hline São José do Rio Preto & 95 & 75.878 & 1.308 .239 & 4 \\
\hline Rio de Janeiro & 94 & 53.006 & 14.229 .857 & 6 \\
\hline Bauru & 86 & 32.591 & 1.680 .688 & 6 \\
\hline Vitória & 80 & 58.942 & 3.298 .912 & 4 \\
\hline
\end{tabular}

Na atenção hospitalar de alta complexidade, a primeira rede a ser investigada baseou-se nos fluxos que demandam atendimento em Cirurgia Cardíaca, e está apresentada na Figura 4. No total, 85 municípios internaram pessoas nesta especialidade e destes, 80 internaram pessoas residentes fora de seus limites. Registre-se, além disso, que 3.004 municípios não enviaram pacientes para tratamento. Considerando a distribuição de pacientes em todos os pares município de residência-município de internação, 60,0\% dos fluxos correspondem a menos de cinco pessoas, e $4,0 \%$ a trinta ou mais pessoas; em relação ao total acumulado de pacientes que saem de cada município, esses valores são de $51,0 \%$ e $8,0 \%$, respectivamente.

As internações para Cirurgia Cardíaca conformaram três redes, as de Aracaju, Itaperuna (Rio de Janeiro) e São Paulo. Aracaju, com 39 municípios, tem rede basicamente limitada ao próprio estado de Sergipe, e não conta com sub-redes. Todos os fluxos desta rede são do tipo hierárquico-ascendente direto. Itaperuna, com 27 municípios, atrai fluxos dos municípios vizinhos, do Rio de Janeiro, Minas Gerais e Espírito Santo, e também não tem sub-redes. Predominam os fluxos hierárquico-ascendentes diretos, com $74,3 \%$, seguidos pelos transversais, com $25,7 \%$ do total.

São Paulo polariza a rede nacional, com 2.437 municípios. Conta com 272 centros de nível 2, dos quais 87,5 \% não têm subordinados. O mapa da Figura 4 indica que a maior parte das redes de nível 2 é dominada pelas capitais estaduais e centros regionais paulistas e, de modo geral, não ultrapassam as fronteiras estaduais. Como exceção aparece Goiânia, que embora comande região menor do que no caso das redes de atendimento básico, influencia ainda uma grande área. A região comandada por Campo Grande também ultrapassa largamente os limites estaduais, estendendo-se por Mato Grosso e Rondônia. O nível 3 corresponde às redes de centros regionais e demais capitais. A maior parte dos fluxos é do tipo hierárquico direto $72,3 \%$, mais $9,2 \%$ em "curto circuito" no mesmo ramo da rede. As relações transversais não ultrapassam 18,1\%, sendo $16,9 \%$ ascendentes.

Nos 2.500 municípios abaixo do primeiro nível, 86,8\% das pessoas internadas residem a menos de $30 \mathrm{~km}$ do município do hospital, e $10,2 \%$ num raio de 30 a $60 \mathrm{~km}$.

A segunda rede de alta complexidade investigada baseou-se nos fluxos que demandam 
Figura 4

Redes de cirurgia cardíaca.

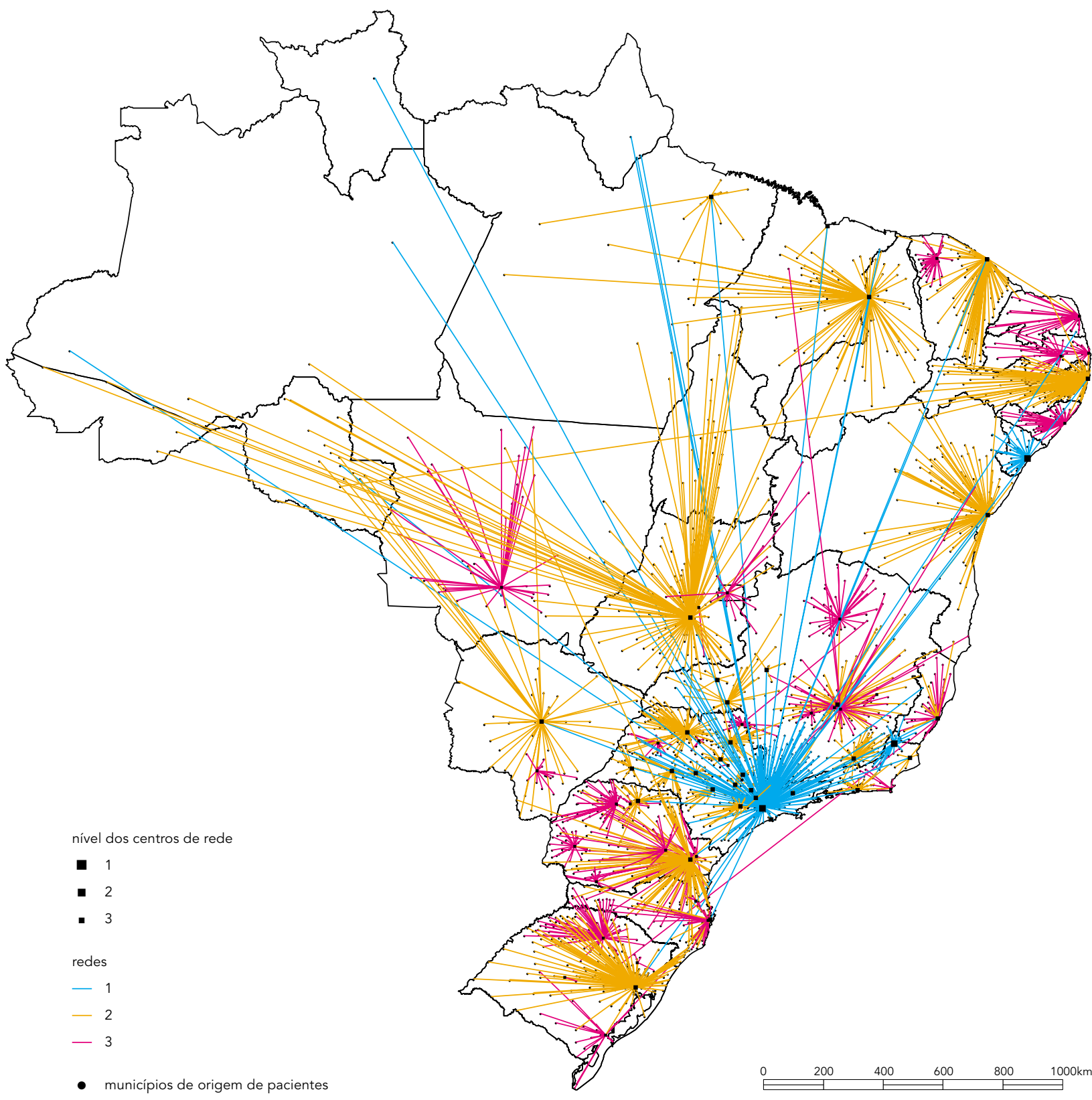


atendimento em Neurocirurgia - Alta Complexidade, e está apresentada na Figura 5. No total, 145 municípios internaram pessoas nesta especialidade e destes, 139 municípios internaram pessoas residentes fora de seus limites. Registre-se, além disso, que 2.386 municípios não enviaram pacientes para tratamento. Considerando a distribuição de pacientes em todos os pares município de residência-município de internação, 53,0\% dos fluxos correspondem a menos de cinco pessoas, e 5,0\% a trinta ou mais pessoas; em relação ao total acumulado de pacientes que saem de cada município, esses valores são de $41,0 \%$ e $7,0 \%$, respectivamente.

As redes relativas às internações para Neurocirurgia - Alta Complexidade são 18, apresentando maior distribuição no território nacional que no caso anterior. A diferença de tamanhos entre as 18 redes de primeiro nível, apresentadas na Figura 5, é flagrante, variando de São Paulo, com 1.435 municípios, Curitiba, Belo Horizonte e Porto Alegre, de 357 a 309, Recife e Florianópolis com 139 e 116, até Rio Bonito (Rio de Janeiro) e Mogi Guaçu (São Paulo), cada qual com três municípios subordinados. A rede de São Paulo conta com 55 centros no segundo nível, dos quais $58,2 \%$ não têm rede própria. Entre os 23 centros que comandam sub-redes estão, além das capitais e centros regionais paulistas, Londrina, Uberlândia (Minas Gerais) e Itabuna (Bahia). Os centros de nível 2 contam com redes mais densas, valendo observar-se a concorrência entre Goiânia, Campo Grande, e até mesmo Brasília, no atendimento dos municípios do noroeste do país.

A maior parte dos fluxos é do tipo hierárquico, direto $(76,3 \%)$, ou em "curto circuito" $(7,5 \%)$. As relações transversais representam $15 \%$ do total, sendo $10,7 \%$ ascendentes.

Nos 3.115 municípios abaixo do primeiro nível, $36,7 \%$ das pessoas internadas residem a menos de $30 \mathrm{~km}$ do município do hospital, e $20,9 \%$ num raio de 30 a $60 \mathrm{~km}$.

O exame da classificação dos fluxos nas quatro redes investigadas (Tabela 5) patenteia os diferentes padrões de organização em cada uma delas. A oposição entre as redes de atenção hospitalar básica e as de alta complexidade expressa-se, nas primeiras, pela predominância dos fluxos transversais, que ocorrem entre diferentes ramos; nas demais, predominam os fluxos hierárquicos. Note-se, além disso, que os fluxos hierárquicos são ascendentes, o que quer dizer que municípios de nível mais baixo se dirigem para municípios de nível superior, praticamente não ocorrendo as inversões classificadas como "contra-hierárquicas", ou fluxos descendentes. A cadeia hierárquica também tende a ser obedecida, e só nas redes da população de cinco anos e mais de idade é que a proporção de fluxos em "curto circuito" - aqueles que saltam um ou mais níveis e vão diretamente para um município de posição mais elevada - têm participação mais semelhante à dos fluxos ascendentes diretos.

\section{Discussão}

Os resultados deste estudo evidenciam que cerca de $75,0 \%$ de todas as internações realizadas no país no ano de 2000 ocorreram no município de residência dos pacientes, o que não surpreende ao considerar-se que $91,0 \%$ da população brasileira mora em municípios com hospitais conveniados ao SUS. As diferenças entre as redes de atenção hospitalar básica e as de alta complexidade e, em cada tipo, dos exemplos selecionados, permitem compreender melhor o significado destes valores.

Assim, os diferentes tipos de redes observados demonstram a desigualdade regional no acesso, que é ainda mais marcada em relação aos serviços mais complexos. Ainda que, ao longo dos anos, a efetiva implantação da universalização do acesso aos serviços de saúde, meta do SUS, tenha sido tolhida pelas condições político-econômicas mais gerais, é indiscutível o avanço do setor saúde, no Brasil, em termos de ampliação da cobertura e níveis de atendimento. Entretanto, embora alguns estudos apontem tendência à redução das desigualdades regionais no sistema de saúde 18,19, esse processo é muito lento, pois "a distribuição territorial de infra-estrutura de recursos reforça as desigualdades no acesso aos serviços e na quantidade e qualidade da atenção recebida pela população" 20 (p. 291) e, como observa Santos 21, a geografia dos fluxos depende da geografia dos fixos.

Assim, a atenção hospitalar básica está disponível em praticamente todo o território nacional e poucos são os municípios desconectados. Na primeira rede a predominância dos fluxos transversais destaca a possibilidade de alternativas em relação ao local de atendimento, apontando as interconexões entre os vários ramos das redes, o que indica maior difusão do acesso a estas redes. Em relação às redes definidas com base no atendimento das crianças, a divisão dos fluxos em transversais e hierárquicos indica um padrão misto, em que o atendimento é complementado por opções fora da rede hierárquica. Destacam-se aqui, além da ampla cobertura, as pequenas distâncias, exce- 
Figura 5

Redes de neurocirurgia - alta complexidade.

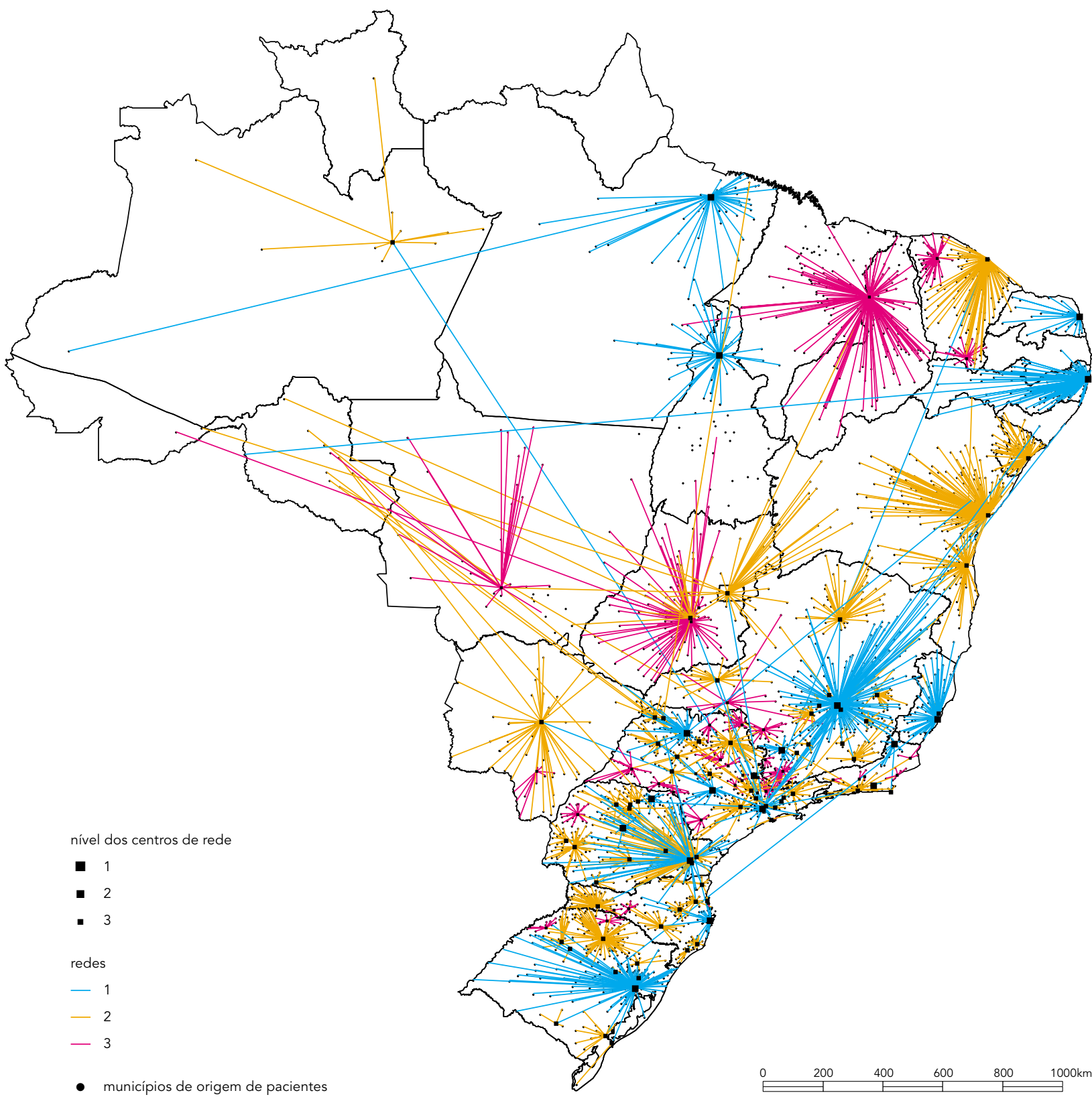


Classificação dos fluxos nas redes investigadas. Brasil, 2000.

\begin{tabular}{lcccc}
\hline Tipos de fluxos & $\begin{array}{l}\text { População de } \\
\text { cinco e mais anos } \\
\text { de idade (\%) }\end{array}$ & Crianças (\%) & $\begin{array}{l}\text { Cirurgia } \\
\text { cardíaca (\%) }\end{array}$ & $\begin{array}{c}\text { Neurocirurgia - alta } \\
\text { complexidade (\%) }\end{array}$ \\
\hline Transversais & 60,8 & 40,6 & 18,0 & 15,4 \\
Hierárquico-ascendentes & 34,9 & 56,4 & 81,6 & 83,8 \\
Diretos & 18,1 & 41,5 & 72,6 & 76,3 \\
Em "curto circuito" & 16,8 & 14,9 & 9,0 & 7,5
\end{tabular}

to nas áreas de povoamento mais rarefeito, onde aumenta a extensão a percorrer entre os municípios de residência e o do hospital. Para as pessoas de cinco anos e mais de idade, registram-se atendimentos em locais mais - às vezes muito - distantes, fato que pode estar ligado à estrutura das relações ao longo da rede urbana, e não necessariamente à carência de recursos locais. Vale dizer que as pessoas viajam - a trabalho ou para lazer - e podem ser surpreendidas por problemas de saúde que exijam atendimento de emergência fora do município de residência.

Situação bem diversa é a das redes de alta complexidade, nas quais apenas um pequeno número de centros presta atendimento, e cerca de metade dos municípios brasileiros está desconectada: $54,5 \%$ no caso da Cirurgia Cardíaca, e $43,3 \%$ no caso da Neurocirurgia - Alta Complexidade. As duas redes investigadas têm padrão de fluxos marcadamente hierárquico, e são poucas as ligações estabelecidas fora do arcabouço das redes. Esse é, de resto, um dos mecanismos clássicos de racionamento e restrição da demanda por serviços escassos. Apesar destas semelhanças, o desenho das duas redes é bem diferente, sendo a primeira polarizada quase que exclusivamente por São Paulo, enquanto a segunda tem padrão de distribuição mais descentralizado. Tal fato reflete a diferença dos dois tipos de tratamento, o primeiro mais programado, o segundo muito ligado a situações de emergência, decorrentes de causas externas e doença cérebro-vascular.

O método utilizado não é capaz de superar uma limitação decorrente da instabilidade das ligações determinadas por fluxos de pequeno número de pessoas. De fato, a flutuação decorrente da menor freqüência pode explicar alguns casos de municípios sem atendimento, que não dispõem de hospital conveniado e não internaram pacientes em outros municípios. Numa tentativa de garantir maiores volumes, optou-se por agregar as internações de todo o ano de 2000. No caso dos procedimentos de alta complexidade, entretanto, o volume realizado no período de um ano é ainda relativamente pequeno, sendo muito provável que o emprego de informações de outros anos apresente resultados diversos dos aqui expostos, ainda que a estrutura básica se mantenha e as alterações afetem mais as extremidades das redes. Estudos futuros deverão explorar maneiras de eliminar, ou reduzir, este problema.

A interpretação da hierarquia das redes identificadas exige cautela. Recorde-se que o método empregado garante somente que - ao longo de um mesmo ramo da rede - o menor centro estará subordinado a um de maior tamanho, e que essas relações de subordinação são transitivas. Assim, um pequeno centro periférico ligado apenas a um único vizinho poderá estar incluído na área de um polo de expressão nacional, ainda que não tenha qualquer relação direta com o mesmo. Um exemplo ilustrativo é o do Município de Tabatinga (Amazonas), na fronteira com a Colômbia, que na rede das pessoas de cinco anos e mais é um centro de nível 3 da rede de São Paulo, diretamente ligado a Manaus, centro de nível 2. Dependendo da extensão da cadeia envolvida, municípios de porte ou complexidade semelhantes poderão ocupar níveis bem diversos. Numa área onde os objetivos de regionalização e hierarquização do atendimento já estejam mais avançados, vários níveis hierárquicos estarão presentes; em outras áreas, a maior parte dos municípios contará com apenas uma opção de nível superior. Desta forma, o estudo da hierarquia da rede não pode ser limitado à consideração dos níveis dos centros em cada ramificação. Centros de mesmo nível em redes diferentes poderão ter características radicalmente díspares entre si, dependendo do perfil de seu superior. Para cirurgia cardíaca, por exemplo, Itaperuna, Aracaju e São Paulo comandam re- 
des independentes, de modo que os municípios de Cordeiro (Rio de Janeiro) e Salgado (Sergipe), com menos de 20 mil habitantes, e o do Rio de Janeiro, entre outros, são centros de nível 2. Nesse sentido, a independência das redes de Itaperuna e Aracaju traduz a falta de fluxos de sua população para centros de outras redes. Mesmo considerando uma única rede, os centros de segundo nível poderão representar pólos de sub-redes próprias, ou simplesmente estar diretamente ligados ao nível 1. Assim sendo, muitos dos fluxos transversais classificados como horizontais (ou até mesmo como descendentes) dirigem-se, na verdade, para centros maiores e melhor equipados do que o centro que constitui o nível superior.

O método de mapeamento das redes aqui empregado limita-se a investigar as ligações definidas pela ausência ou presença de determinado serviço, e não é capaz de dimensionar a oferta ou a demanda, não avaliando taxas de atendimento ou questões de necessidade. É certamente possível que a prevalência das condições investigadas seja desigualmente distribuída. Não se pode, entretanto, descartar a indicação de problemas de acesso, ao comparar-se, no caso da atenção básica, as distâncias dos municípios que, estando conectados à rede quando se considera a população de cinco anos e mais de idade, não internaram qualquer criança, e acham-se situados a grandes distâncias dos centros de atendimento, configurando vastas áreas desassistidas.

Em relação ao efeito da distância no acesso aos serviços, é fato largamente estabelecido na literatura, e mesmo no dia a dia, que o raio de alcance dos serviços de mais alto nível é maior que o dos serviços básicos: as pessoas irão mais longe para obter bens e serviços mais complexos. No caso das redes de alta complexidade aqui investigadas, a concentração do atendimento em poucos centros determina a existência de maiores distâncias a percorrer para obter atendimento. O que ocorre é que, de fato, as pessoas que vivem mais longe não têm a possibilidade de deslocar-se: apenas 3,0\% dos pacientes internados para cirurgia cardíaca residiam em municípios situados a mais de $60 \mathrm{~km}$ do centro em que se internaram, e nestes municípios concentram-se cerca de $40,0 \%$ da população brasileira. Compare-se esta situação ao que ocorre nas internações nos procedimentos mais freqüentes: pouco menos de $20,0 \%$ dos pacientes de cinco anos e mais de idade internaram-se em centros a mais de $60 \mathrm{~km}$, mas as pessoas residentes além deste limite representam apenas 1,3\% da população total. Importa ressaltar que este trabalho investigou apenas a estrutura da rede, sem dimensionar a oferta e a demanda, aspectos importantes da questão do acesso. Considerados esses resultados à luz das pretensões expressas na NOAS, verifica-se que o seu objetivo de garantir o acesso aos serviços de saúde de uso mais freqüente, o mais próximo possível da residência dos cidadãos, parece, se não alcançado, alcançável no curto prazo. Se, porém, a oportunidade de receber tratamento deve refletir a necessidade de saúde da população e não a distância do serviço 22 , a organização das referências intermunicipais para os outros níveis de atenção ainda tem muito que avançar.

\section{Resumo}

Este trabalho investiga dois tipos de redes estabelecidas pelos fluxos de pacientes para os serviços de saúde: a de atenção hospitalar básica, definida pelos procedimentos com maior freqüência de internação e as de atenção de alta complexidade.Agregaram-se os dados do Sistema de Informações Hospitalares do Sistema Único de Saúde sobre as internações realizadas no ano de 2000 em todo o Brasil-segundo o município de internação e o de residência dos pacientes. Empregou-se o método do fluxo dominante, que define o arcabouço da rede e os níveis hierárquicos dos municípios que constituem os nós. A classificação dos fluxos avalia o grau de interconexão das redes. As redes de atenção hospitalar básica alcançam quase todo o país; poucos municípios estão fora delas. Nas redes de alta complexidade poucas cidades prestam atendimento e cerca da metade dos municípios brasileiros está desconectada. O objetivo da Normas Operacionais de Atenção à Saúde, de garantir o acesso aos serviços de saúde de uso mais freqüente perto da residência dos cidadãos, parece alcançável em curto prazo. A organização das referências para os outros níveis de atenção, entretanto, ainda tem muito que avançar.

Acesso aos Serviços de Saúde; Atenção; Serviços de Saúde 


\section{Colaboradores}

E. X. G. de Oliveira participou da concepção da pesquisa, análise e processamento dos dados e redação do artigo. M. S. Carvalho e C. Travassos contribuíram na concepção da pesquisa, análise dos dados e redação do artigo.

\section{Referências}

1. Ministério da Saúde. Regionalização da assistência à saúde: aprofundando a descentralização com eqüidade no acesso - Norma Operacional de Assistência à Saúde 01/01. Brasília: Ministério da Saúde; 2001.

2. Knoke D, Kuklinski JH. Network analysis: basic concepts. In: Thompson G et al., organizadores. Markets, hierarchies and networks. London: Sage Publications; 1991.p. 173-82.

3. Bakis R. Les réseaux et les enjeux sociaux. Paris: Presses Universitaires de France; 1993.

4. Donabedian A. Explorations in quality assessment and monitoring. The definition of quality and approaches to its assessment. Ann Arbor: Health Administration Press; 1980.

5. Penchansky R, Thomas, JW. The concept of access: definition and relationship to consumer satisfaction. Med Care 1981; 19:127-40.

6. Frenk J. El concepto y la medición de la accessibilidad. Salud Pública Méx 1985; 27:438-53.

7. Kane R. Determinants of health care priorities and expectations among rural consumers. Health Serv Res 1969; 4:142-51.

8. Mayer JD. The distance behavior of hospital patients: a disaggregated analysis. Soc Sci Med 1983; 17:819-27.

9. McGuirk MA, Porell FW. Spatial patterns of hospital utilization: the impact of distance and time. Inquiry 1984; 21:84-95.

10. Departamento de Informática do SUS. Movimento de Autorização de Internação Hospitalar. Resumo anual 2000. [CD-ROM]. Brasília: Ministério da Saúde; 2000.

11. Ministério da Saúde. Portaria SAS n. 96 de 27 de março de 2000. Aprovar os procedimentos constantes dos anexos I e II desta Portaria, como integrantes dos Sistemas de Alta Complexidade Ambulatorial e Hospital do Sistema Único de Saúde: Anexo I e II. http://dtr2001.saude.gov. $\mathrm{br} / \mathrm{sas} / \mathrm{PORTARIAS} / \mathrm{PORT} 2000$ / anexoii-PT 096.html (acessado em 20/Jan/2003).

12. Ministério da Saúde. Portaria SAS n. 579 de 28 de dezembro de 2001. Art. 1o - Aprovar a tabela de compatibilidade entre o procedimento realizado e o diagnóstico principal informado, de acordo com a classificação estatística internacional de doenças e problemas relacionados à saúde - décima revisão no processamento das AIH - Autorização de Informação Hospitalar do SIH - Sistema de Informações Hospitalares. http://dtr2001.saude. gov.br/sas / PORTARIAS / Port2001/PT-579.htm (acessado em 20/Jan/2003).

13. Diestel R. Graph theory graduate texts in mathematics. Verlag: Springer; 1997.

14. Nystuen JD, Dacey MF. A graph theory interpretation of nodal regions. Papers and Proceedings of the Regional Science Association 1961; 7:29-42.

15. Taaffe EJ, Gauthier HL. Geography of transportation. Englewood Cliffs: Prentice-Hall; 1973.

16. Rabino GA, Occelli S. Understanding spatial structure from network data: Theoretical considerations and applications 1997; Cybergeo 29. http:// 193.55.107.45/reseaux/texte1/gron1.htm (acessado em 08/Nov/2002).

17. Mabini L, Rabino G. Fluplite ver. 1.01c. 1995; http:// www.diap.polimi.it/ grabino/metodiemodelli/ Gerarchie/gerarchie1_en.htm (acessado em 08/ Nov/2002):

18. Almeida C, Travassos C, Porto S, Labra ME. Health sector reform in Brazil: a case study of inequity. Int J Health Serv 2000; 30:129-62.

19. Travassos C, Viacava F, Fernandes C, Almeida CM. Desigualdades geográficas e sociais na utilização de serviços de saúde no Brasil. Ciên Saúde Coletiva $2000 ; 5: 133-49$.

20. Teixeira CM. Epidemiologia e planejamento de saúde. Ciên Saúde Coletiva 1999; 4:287-303.

21. Santos M. A natureza do espaço: técnica e tempo; razão e emoção. 2a ed. São Paulo: Hucitec; 1999.

22. Haynes R, Bentham G, Lovett A, Gale S. Effects of distances to hospital and GP surgery on hospital inpatient episodes, controlling for needs and provision. Soc Sci Med 1999; 49:425-33.

Recebido em 17/Fev/2003

Versão final reapresentada em 16/Set/2003

Aprovado em 24/Set/2003 